\title{
Spectroscopic characterization of insulin and small molecule ligand binding to the insulin receptor
}

\author{
Morten Schlein ${ }^{\mathrm{a}, *}$, Svend Ludvigsen ${ }^{\mathrm{a}}$, Helle B. Olsen ${ }^{\mathrm{a}}$, Michael F. Dunn ${ }^{\mathrm{b}}$ and \\ Niels C. Kaarsholm ${ }^{\text {a }}$ \\ ${ }^{a}$ Research \& Development, Novo Nordisk A/S, Novo Alle 1, DK-2880 Bagsvaerd, Denmark \\ ${ }^{\mathrm{b}}$ Department of Biochemistry, University of California at Riverside, Riverside, CA 92521, USA
}

\begin{abstract}
We have applied spectroscopic techniques to study two kinds of ligand binding to the insulin receptor. First, a fluorescently labelled insulin analogue is used to characterize the mechanism of reversible $1: 1$ complex formation with a fragment of the insulin receptor ectodomain. The receptor induced fluorescence enhancement of the labelled insulin analogue provides the basis for stopped flow kinetic experiments. The kinetic data are consistent with a bimolecular binding event followed by a conformational change. This emphasizes the importance of insulin induced conformational changes in the activation of the insulin receptor. Second, the binding of fluorescein derivatives to the insulin receptor is studied. These small molecule ligands displace insulin from its receptor with micromolar affinity. The binding is verified by transferred NOESY NMR experiments. Their chromophoric properties are used to measure the affinity by UV-vis and fluorescence difference spectroscopies and the resulting $K_{\mathrm{d}}$ values are similar to those observed in the displacement receptor binding assay. However, these experiments and a stoichiometry determination indicate multiple binding sites, of which one overlaps with the insulin binding site. These two examples illustrate how spectroscopy complements biochemical receptor binding assays and provides information on ligandinsulin receptor interactions in the absence of three dimensional structures.
\end{abstract}

\section{Introduction}

The insulin-insulin receptor (IR) system is a thoroughly investigated hormone-receptor pair [46]. Its role in glucose regulation and metabolism in general is well known and its dysfunction leads to diabetes [47].

The lack of crystal structures of IR and/or its complex with insulin emphasizes the importance of solution studies of the hormone-receptor interaction. Furthermore, suggested improvements of diabetes treatment, e.g., small molecule insulin mimetics, motivate the study of ligand binding to IR. In both cases, the application of a repertoire of spectroscopic techniques proves useful.

The insulin molecule (see Fig. 1A) consists of 21 residues in an A-chain and 30 residues in a B-chain. Two disulfide bridges connect the two chains, and a third disulfide bridge is found internally in the A-chain. The understanding of insulin structure-activity relationships is based upon X-ray crystallography [3] and nuclear magnetic resonance (NMR) spectroscopy of monomeric, biological active insulin analogues $[27,28,35,36]$.

IR is organized as a heterotetramer consisting of two $\alpha-\beta$ subunits, see Fig. 1B [45]. The subunits are connected by disulfide bridges [44]. The $\alpha$ subunit is extracellular and contains the insulin binding

\footnotetext{
${ }^{*}$ Corresponding author. Tel.: +45 4442 6146; Fax: +45 4444 4565, +45 4444 4256; E-mail: mosc@ @ovonordisk.com.
} 


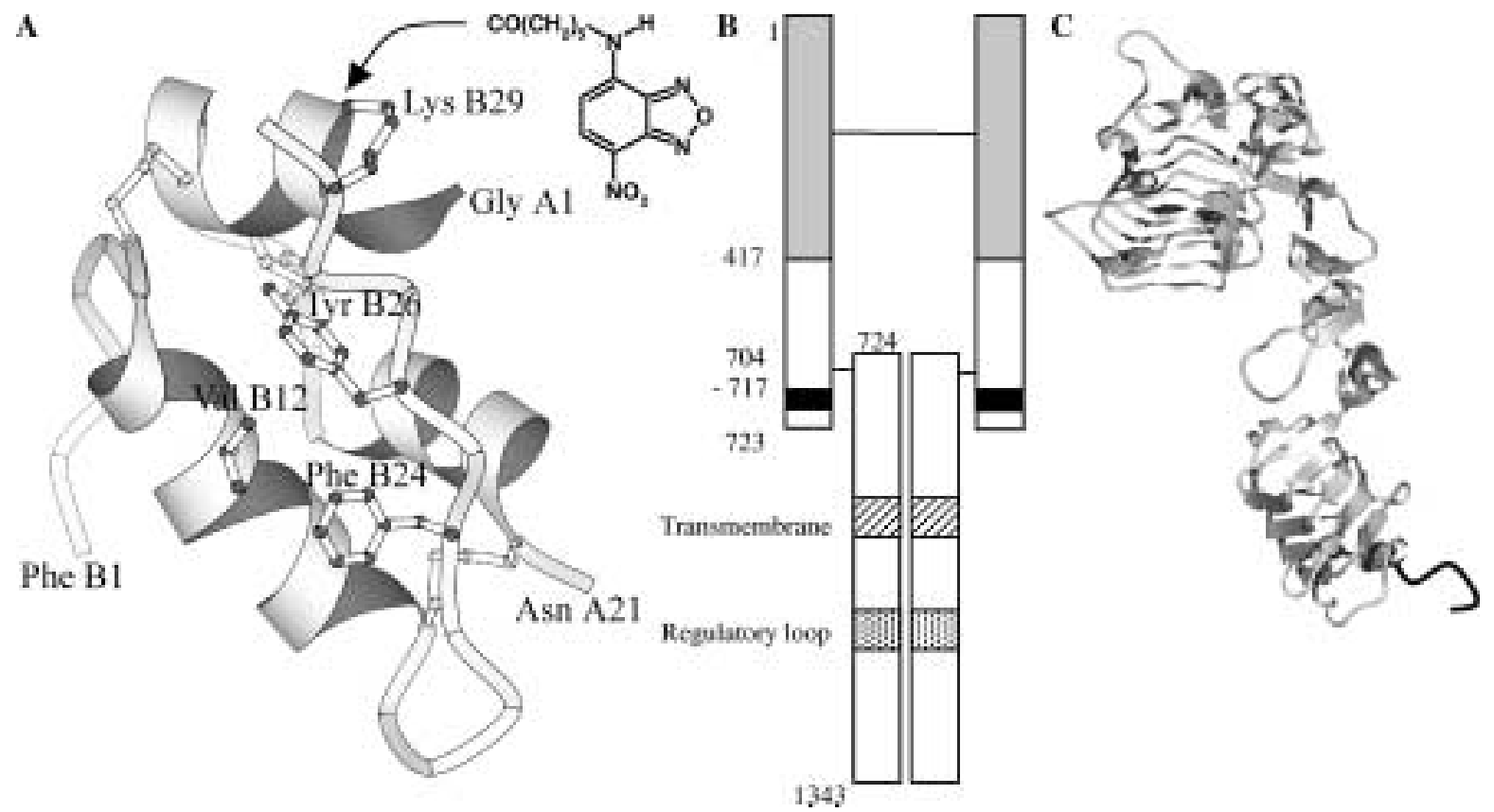

Fig. 1. Insulin and the insulin receptor. (A) Structure of insulin (1hls.pdb). LysB29 is the attachment point for the NDB probe shown right. ValB12, PheB24 and TyrB26 form an epitope important for receptor binding. The plot was generated using the Molscript program [21]. (B) Schematic representation of the insulin receptor. The $\alpha$ subunit consists of residues 1-723, the $\beta$ subunit of 724-1343 (numbering according to [45]). Disulfide bridges are shown as vertical black lines. The transmembrane segment and the regulatory loop of the tyrosine kinase domain are indicated. The heterotetrameric sIR is truncated at residue 917. The 1-417 segment (grey) together with the 704-717 segment (black) constitute the monomeric mIR. (C) Structure of an IGF-1 receptor fragment (1igf.pdb). The part corresponding to residues 1-417 in mIR is shown in grey. The residues 704-717, conferring ligand binding in $\mathrm{mIR}$, are drawn as a black line attached to the $\mathrm{C}$-terminal.

domain(s). The $\beta$ subunit has a short extracellular part, a transmembrane segment, and an intracellular part containing a tyrosine kinase domain, of which the structure is known [16,17]. In response to insulin binding, a regulatory loop containing three tyrosines in the kinase domain becomes autophosphorylated [38] bringing the loop to an active conformation with significantly increased kinase activity towards intracellular signalling molecules [7,9]. However, the activation loop conformation in vivo likely depends on the interaction with ATP $[1,11]$.

A number of truncated receptor constructs have been made. The soluble insulin receptor (sIR) is truncated in front of the transmembrane segment at position 917 (numbering excluding exon 11) [2]. Thus, it contains the ligand binding part in a heterotetrameric structure but excludes the tyrosine kinase domain. For immobilization in microtiter plates, sIR has been fused to the $\mathrm{Fc}$ domain of immunoglobulin $\mathrm{G}$ (sIRFc). The minimized insulin receptor (mIR) is the minimal part of sIR capable of binding insulin without loss of affinity [24]. This monomeric construct consists of residues 1-468 and 704-717 of the $\alpha$ subunit. The residues 1-468 correspond to the part of the homologous insulin-like growth factor-1 (IGF-1) receptor of which a crystal structure has been solved, see Fig. 1C [12]. A major part of the binding affinity is conferred by the 707-714 sequence [23]. This sequence even appears capable of restoring binding activity of the 1-486 fragment when added as a peptide in large excess [22]. Consequently, the structure of the minimized IGF-1 receptor construct does not bind its ligand [12].

We have recently applied various spectroscopic techniques to study ligand binding to IR in two cases. 
First, insulin was labelled with the fluorophore succinimidyl 6-(N-(7-nitrobenz-2-oxa-1,3-diazol-4yl)amino)hexanoate (NBD) at position LysB29. No loss of binding was observed due to the labelling, i.e., its binding affinity towards $\mathrm{mIR}$ was $K_{\mathrm{d}}=11 \pm 6 \mathrm{nM}$ compared to $K_{\mathrm{d}}=18 \pm 4 \mathrm{nM}$ for human insulin. The binding of the B29NBD-insulin derivative to mIR was followed by both static and stoppedflow kinetic fluorescence measurements. This enabled us to propose a mechanism for the binding which consists of a fast bimolecular association followed by a slower unimolecular process most likely involving a conformational change in the intermediate insulin-receptor complex [40].

Second, the binding of small molecule insulin antagonists to IR has been studied. These ligands were found in an attempt to mimic the insulin epitope consisting of ValB12, PheB24 and TyrB26. This epitope is thought to be important for receptor binding ([27] and references herein), see Fig. 1A. From database searches for molecules mimicking this epitope and subsequent biochemical analysis, thymolphthalein was found to partially displace insulin from IR and weakly stimulate IR in a number of assays. These effects occurred in the concentration range of $10 \mu \mathrm{M}-1 \mathrm{mM}$. A second database search followed by biochemical analysis showed that analogues of the related phenol red and fluorescein molecules fully displaced insulin from IR, however with antagonistic effect. Two of the best hits were eosin (EO; $K_{\mathrm{d}}=$ $40 \pm 4 \mu \mathrm{M})$ and erythrosine (ER, $\left.K_{\mathrm{d}}=11 \pm 1 \mu \mathrm{M}\right)$ [41]. The spectroscopic characterization of these ligands is discussed herein.

\section{Materials and methods}

The experimental details are described elsewhere [40,41].

The fluorescence enhancement of B29NBD-insulin was fitted to the following one-site binding equation:

$$
\Delta F=F_{\max } \frac{\left([\mathrm{P}]+[\mathrm{L}]+K_{\mathrm{d}}\right)-\sqrt{\left([\mathrm{P}]+[\mathrm{L}]+K_{\mathrm{d}}\right)^{2}-(4[\mathrm{P}][\mathrm{L}])}}{2[\mathrm{P}]} .
$$

The fluorescence time courses obtained by stopped-flow techniques were fitted to the sum of exponentials following Eq. (2):

$$
F_{t}=F_{\infty} \pm \sum F_{i} \exp \left(-t / \tau_{i}\right)
$$

Spectroscopic determination of the $K_{\mathrm{d}}$ values for the small molecule ligands were performed under conditions were Eq. (1) could be expressed as:

$$
\Delta=\Delta_{\max } \frac{[\mathrm{L}]}{[\mathrm{L}]+K_{\mathrm{d}}},
$$

where $[\mathrm{L}]$ is the total ligand concentration.

The Job's plot was constructed by depicting the difference between two extremes of $\Delta \mathrm{Abs}$ as a function of the varying mole fraction. The tangents to this curve was drawn through $x=0$ and $x=1$. The mole fraction $x_{\mathrm{s}}$ at the intersect of these were used to calculate the stoichiometry, $n$, using Eq. (4) [15].

$$
n=\frac{1-x_{\mathrm{s}}}{x_{\mathrm{s}}}
$$



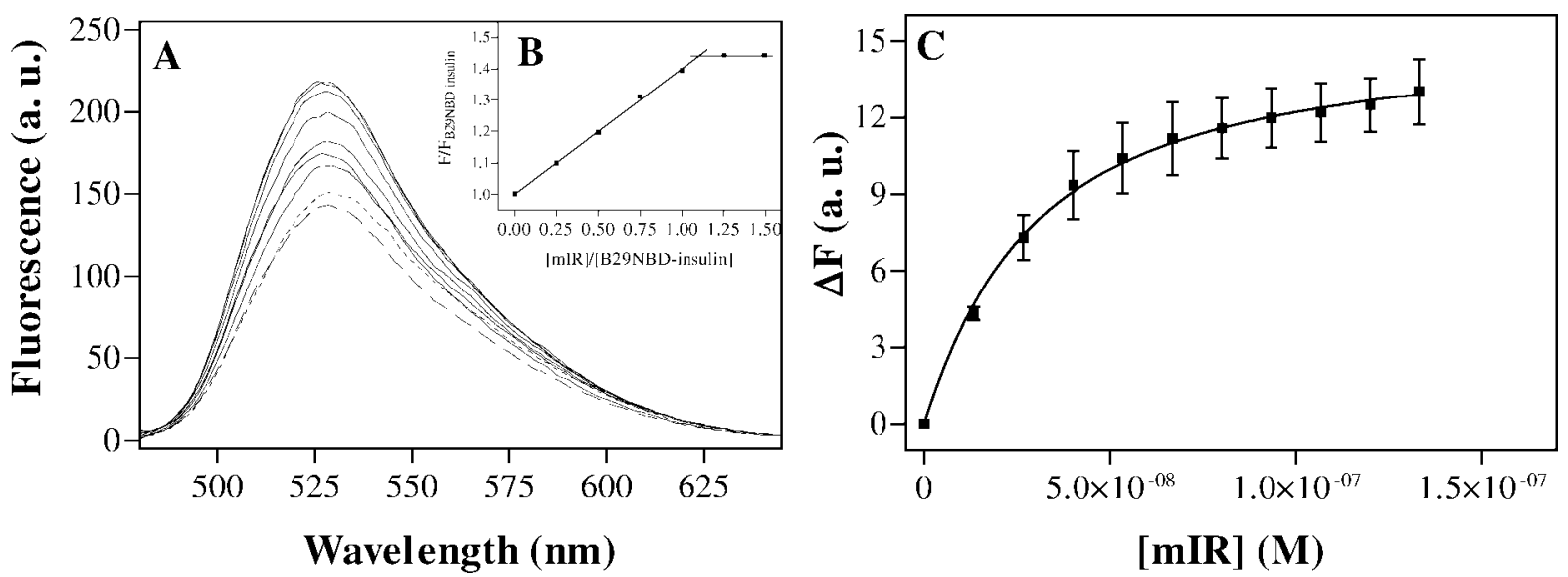

Fig. 2. Fluorescence change of B29NBD-insulin in the presence of mIR. (A) Emission spectra of $0.7 \mu \mathrm{M}$ B29NBD-insulin excited at $470 \mathrm{~nm}$ in the absence of $\mathrm{mIR}$ (dotted line) and the increases in fluorescence resulting from the addition of $0.17,0.33$, 0.5, 0.67, 0.84 and $1 \mu \mathrm{M}$ mIR (solid lines). The dashed line shows the spectrum after equilibrium has been reached following subsequent addition of $30 \mu \mathrm{M}$ HB10D, PB28D mutant insulin to the sample containing $1 \mu \mathrm{M}$ mIR. (B) Relative fluorescence enhancement at $530 \mathrm{~nm}$ as a function of added mIR for the titration in A. (C) Fluorescence increase at $530 \mathrm{~nm}$ of $10 \mathrm{nM}$ B29NBD-insulin excited at $470 \mathrm{~nm}$ with increasing mIR concentration. Data points were fitted to a one-site binding model (Eq. (1)) resulting in a binding curve (full line) with $K_{\mathrm{d}}=23 \pm 6 \mathrm{nM}$. SEM bars are included. Reprinted with permission from Journal of Molecular Biology 303 (2000), 161-169. Copyright 2000 Academic Press.

\section{Results}

\subsection{Binding of a fluorescently labelled insulin analogue to $\mathrm{mIR}$}

The $530 \mathrm{~nm}$ fluorescence emission band of free B29NBD-insulin was enhanced approximately $40 \%$ and blue shifted 1-2 $\mathrm{nm}$ when binding to $\mathrm{mIR}$, see Fig. 2A. This titration was performed under conditions where the fluorescence enhancement is a linear function of added B29NBD-insulin, until saturation is reached at a stoichiometry of one molecule labelled insulin per one molecule mIR, see Fig. $2 \mathrm{~B}$. The fluorescence enhancement could be fully displaced within minutes by addition of large excess of the unlabelled, monomeric insulin HB10D, PB28D analogue demonstrating full reversibility of the B29NBDinsulin-mIR interaction. In another titration, the affinity was found to be $K_{\mathrm{d}}=23 \pm 6 \mathrm{nM}$ by measuring the mIR induced fluorescence enhancement of $10 \mathrm{nM}$ B29NBD-insulin (Fig. 2C).

The rate of fluorescence enhancement following the binding of B29NBD-insulin by mIR was measured under pseudo first-order conditions in a stopped flow apparatus. The observed kinetic traces were fitted to a biphasic exponential function, see Fig. 3A.

In order to accurately determine the shape of the concentration dependence of the slower relaxation on [B29NBD-insulin], additional time courses were collected under conditions where [B29NBD-insulin] is less than five times [mIR]. However, the pseudo-first-order approximation will become valid as the reaction goes towards completion.

The relaxation rate constants for the two kinetic phases obtained from the biphasic exponential function are plotted as a function of [B29NBD-insulin] in Fig. 4.

The displacement of B29NBD-insulin by an excess of unlabeled insulin HB10D, PB28D was measured in a conventional fluorimeter, see Fig. 3B. These time courses were fitted to a monophasic exponential function and the rate was found to be $0.01 \mathrm{~s}^{-1}$ independent of both the concentration of the unlabelled 

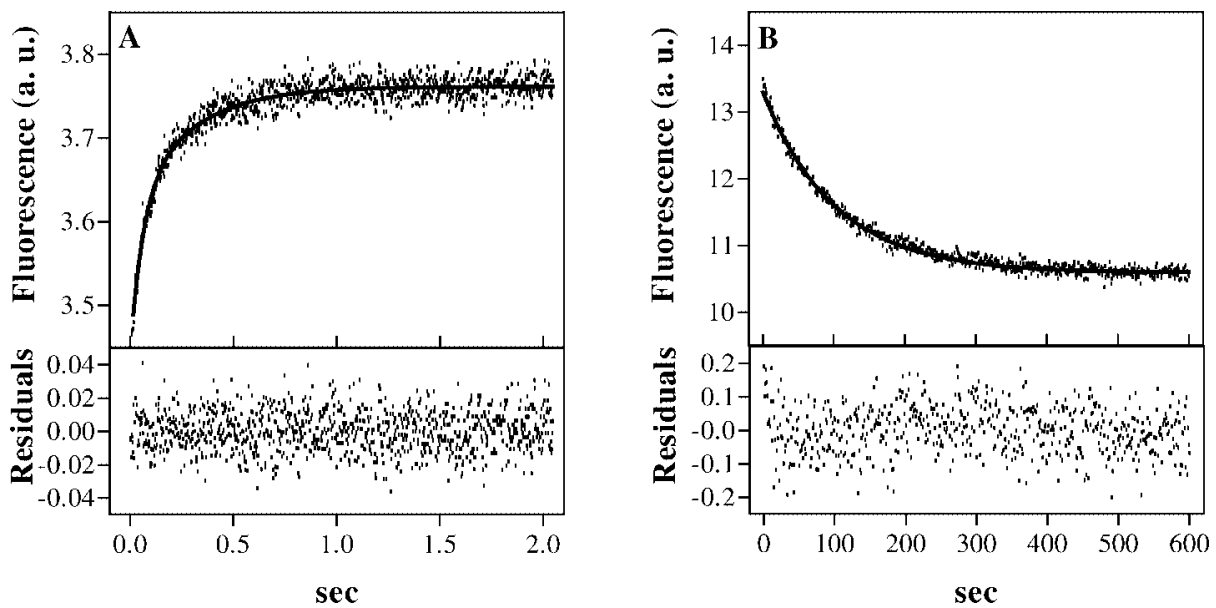

Fig. 3. Representative fluorescence time courses for the formation and dissociation of the B29NBD-insulin-mIR complex. (A) The biphasic increase in fluorescence when $20 \mu \mathrm{M}$ B29NBD-insulin is rapidly mixed with $3 \mu \mathrm{M}$ mIR. The experimental trace is reconstructed by fitting to a biphasic increasing exponential equation (Eq. (2), solid line). Residuals of the fit are shown below. (B) The slow decrease in the $530 \mathrm{~nm}$ fluorescence following the displacement of $0.1 \mu \mathrm{M} \mathrm{B} 29 \mathrm{NBD}$-insulin from $0.2 \mu \mathrm{M}$ mIR by the addition of $1.3 \mu \mathrm{M}$ HB10D, PB28D insulin. The experimental time course is adequately fit by a monophasic decreasing exponential equation (Eq. (2), solid line) as evidenced by the random scatter of the residuals shown below. Reprinted with permission from Journal of Molecular Biology 303 (2000), 161-169. Copyright 2000 Academic Press.

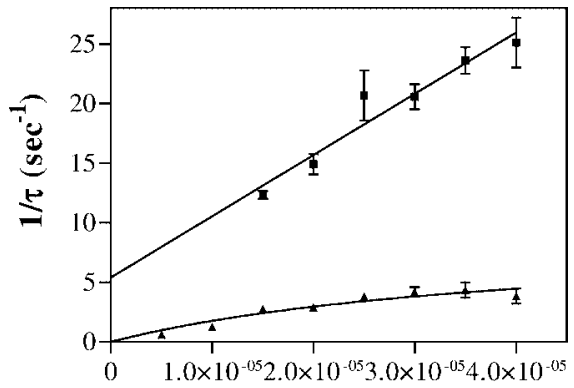

[B29NBD-insulin] (M)

Fig. 4. Pseudo first order rate constants obtained from biphasic exponential fits plotted against B29NBD-insulin concentrations. The fast phase (squares) was fitted to the linear equation: $1 / \tau=k_{1}$ [B29NBD-insulin] $+k_{-1}$. The slow phase (triangles) was fitted to the equation: $1 / \tau=\left(k_{2}\right.$ [B29NBD-insulin] $\left./([\mathrm{B} 29 \mathrm{NBD}-i n s u l i n]+K)\right)+k_{-2}$; where $K=k_{-1} / k_{1}$ and $k_{-2}$ is restrained to $0.01 \mathrm{~s}^{-1}$ as determined separately in the displacement experiments (Fig. 3B). SEM bars included. Reprinted with permission from Journal of Molecular Biology 303 (2000), 161-169. Copyright 2000 Academic Press.

ligand in excess and the B29NBD-insulin concentration. This showed that the displacement reaction is unimolecular and most likely is limited by the dissociation of labelled insulin from mIR.

The simplest plausible mechanism that explains the kinetic data is one that includes a bimolecular binding reaction followed by a unimolecular isomerization step, i.e., $\mathrm{A}+\mathrm{B} \rightarrow \mathrm{C} \rightarrow \mathrm{D}$ as shown in Eq. (5).

B29NBD-insulin $+\mathrm{mIR} \underset{k_{-1}}{\stackrel{k_{1}}{\rightleftharpoons}}[\mathrm{B} 29 \mathrm{NBD}-\mathrm{insulin}-\mathrm{mIR}]$,
[B29NBD-insulin-mIR] $\underset{k_{-2}}{\stackrel{k_{2}}{\rightleftharpoons}}[\mathrm{B} 29 \mathrm{NBD}-\mathrm{insulin}-\mathrm{mIR}]^{*}$. 
Table 1

Parameters for B29NBD-insulin mIR interaction. Reprinted with permission from Journal of Molecular Biology 303 (2000), 161-169. Copyright 2000 Academic Press

\begin{tabular}{ccc}
\hline & & Std. error \\
\hline$k_{1}{ }^{\mathrm{a}}$ & $510,000 \mathrm{M}^{-1} \mathrm{~s}^{-1}$ & 67000 \\
$k_{-1}{ }^{\mathrm{a}}$ & $5.4 \mathrm{~s}^{-1}$ & 1.9 \\
$k_{2}{ }^{\mathrm{b}}$ & $9.1 \mathrm{~s}^{-1}$ & 2.7 \\
$K_{1}{ }^{\mathrm{b}}$ & $41 \mu \mathrm{M}$ & 20 \\
$K_{1}{ }^{\mathrm{c}}$ & $10.6 \mu \mathrm{M}$ & 4 \\
$k_{-2}{ }^{\mathrm{d}}$ & $0.01 \mathrm{~s}^{-1}$ & $2 \times 10^{-4}$ \\
$K_{\mathrm{d}}{ }^{\mathrm{e}}$ & $23 \mathrm{nM}$ & 6 \\
$K_{\mathrm{d}}{ }^{\mathrm{f}}$ & $11 \mathrm{nM}$ & 6 \\
$\left(k_{-1} / k_{1}\right)\left(k_{-2} / k_{2}\right)^{\mathrm{g}}$ & $12 \mathrm{nM}$ & 6 \\
\hline
\end{tabular}

Constants determined from ${ }^{\mathrm{a}}$ linear and ${ }^{\mathrm{b}}$ hyperbolic fits (Fig. 4), ${ }^{\mathrm{c}} k_{-1} / k_{1},{ }^{\mathrm{d}}$ kinetic fluorescence displacement experiments (Fig. 3B), equilibrium fluorescence titration (Fig. 2C) and ${ }^{\mathrm{f}}$ tracer based displacement assay [40]. ${ }^{\mathrm{g}}$ Equals $K_{\mathrm{d}}$.

According to such a model, the concentration dependencies of the fast and slow relaxation rates should be fitted to a straight line and a hyperbolic expression, respectively, see Fig. 4 and Eq. (6) where $K_{1}=$ $k_{-1} / k_{1}[4]$.

$$
\begin{aligned}
& 1 / \tau_{\text {fast }}=k_{1}[\mathrm{~B} 29 \mathrm{NBD}-\text { insulin }]+k_{-1}, \\
& 1 / \tau_{\text {slow }}=\left(k_{2}[\mathrm{~B} 29 \mathrm{NBD}-\text { insulin }] /\left([\mathrm{B} 29 \mathrm{NBD}-\text { insulin }]+K_{1}\right)\right)+k_{-2} .
\end{aligned}
$$

The rate constants obtained from these fit are shown in Table 1 . The overall $K_{\mathrm{d}}$ resulting from this analysis is $12 \mathrm{nM}$, which is equal to the $11 \mathrm{nM}$ value obtained in the displacement binding assay and the $23 \mathrm{nM}$ value obtained in the equilibrium titration (Fig. 2C). We conclude that the observed kinetics are well described by a fast binding step followed by a slower isomerization step that probably involves a conformation change in the B29NBD-insulin-mIR complex.

Circular dichroism (CD) spectra of mIR were collected in the absence and in the presence of 0.8 and $1.6 \mathrm{~mol}$ insulin per mol mIR. In the near UV range, significant changes were apparent as a result of complex formation (Fig. 5). Since no changes were observed in the far UV range, these data are consistent with an insulin induced conformational change involving rigid body movement of structural elements in the receptor fragment.

\subsection{Small molecule ligands for IR}

\subsubsection{Ligand binding to sIR measured by spectroscopy}

The chromophoric properties of EO (see Fig. 6A) and ER (similar structure as EO, but with all Br substituted with I) were exploited to measure the binding affinity towards sIR in solution to complement the tracer-based receptor binding assay with immobilized sIRFc.

The presence of sIR induces changes in the EO absorption spectrum with an increase at $532 \mathrm{~nm}$, a decrease at $510 \mathrm{~nm}$ and an isosbestic point at $525 \mathrm{~nm}$ (Fig. 6B). The isosbestic point together with absorption increase and decrease assure that the differences are real. The absorption decrease at $532 \mathrm{~nm}$ fits Eq. (3) with $K_{\mathrm{d}}=24 \pm 12 \mu \mathrm{M}$, see Fig. 6C. This result is essentially equal to $K_{\mathrm{d}}=40 \pm 4 \mu \mathrm{M}$ obtained in the displacement assay, using sIRFc. 


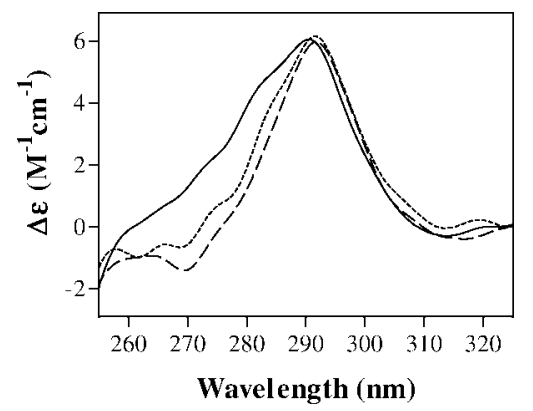

Fig. 5. Effect of insulin on the CD spectrum of mIR in the near UV. The solid line shows the spectrum of $10 \mu \mathrm{M}$ uncomplexed $\mathrm{mIR}$, the dotted line shows the complex with $8 \mu \mathrm{M}$ insulin after subtraction of the contribution from insulin, and the dashed line shows the spectra in the presence of $16 \mu \mathrm{M}$ insulin after subtraction of the insulin contribution. Reprinted with permission from Journal of Molecular Biology 303 (2000), 161-169. Copyright 2000 Academic Press.
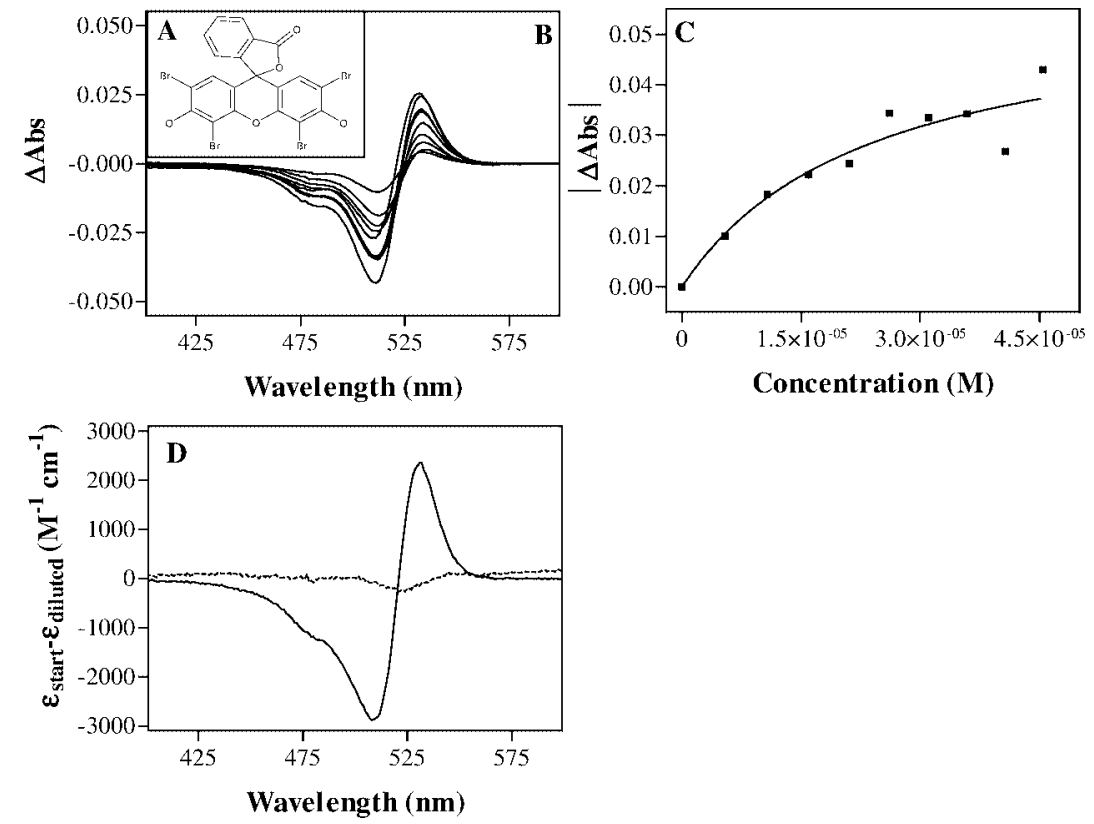

Fig. 6. UV-vis spectroscopy of the EO-sIR interaction. (A) Structure of EO. (B) Difference spectra of eosin in the presence and absence of $2 \mu \mathrm{M}$ sIR. Concentrations of EO in order of increasing difference at $533 \mathrm{~nm}$ are 5.4, 11, 16, 21, 26, 31, 36, 41 and $46 \mu \mathrm{M}$. Spectra were obtained in $100 \mathrm{mM}$ Hepes $\mathrm{pH} 8$ with $0.2 \mathrm{~cm}$ light path. (C) The absorption change at $510 \mathrm{~nm}$ versus [EO]. Full line is Eq. (3) fitted to the data points with $K_{\mathrm{d}}=24 \pm 12 \mu \mathrm{M}$. (D) Dilution of EO-sIR mixture. The difference extinction spectra of $300 \mu \mathrm{M}$ EO alone and in the presence of $2 \mu \mathrm{M}$ sIR under saturating conditions (full line) and the same samples diluted 200 times (broken line).

The reversibility of the EO binding to sIR was checked by obtaining the difference spectrum for a saturating concentration of EO in a sIR containing sample, and comparing this with a spectrum of the same sample diluted 200 times, that is to a ligand concentration lower than $0.1 K_{\mathrm{d}}$. Under these conditions, no difference spectrum occurred between the diluted sample and a sample with a similar concentration of EO alone, see Fig. 6D. Hence, the EO-sIR complex dissociated reversibly.

The ER-sIR affinity was measured by fluorescence spectroscopy. The binding of ER to sIR is accompanied by a small quench of ER emission and a small red shift of the maximum emission wavelength 

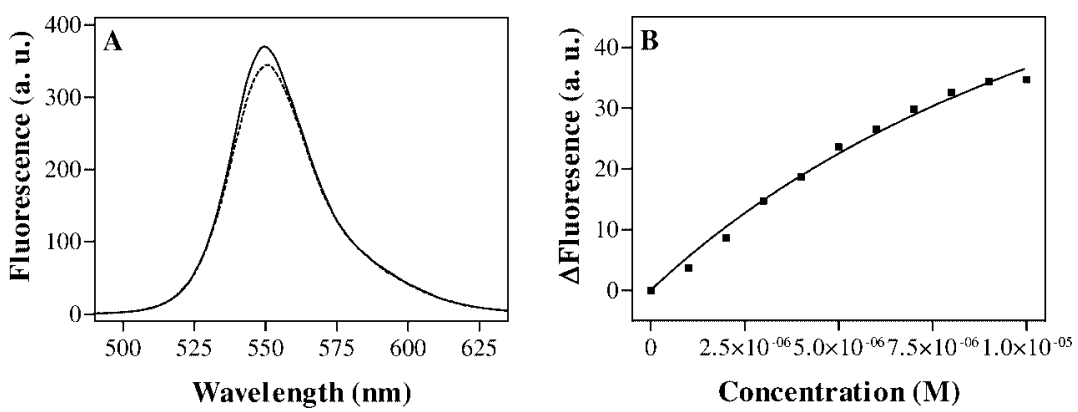

Fig. 7. Fluorescence spectroscopy of ER-sIR interaction. (A) Fluorescence emission spectrum of $10 \mu \mathrm{M}$ ER alone (full line) and with $1 \mu \mathrm{M}$ sIR (broken line) after excitation at $470 \mathrm{~nm}$. Samples prepared in $25 \mathrm{mM}$ Tris pH 8.0. (B) The emission quench of ER concentrations in the presence of $1 \mu \mathrm{M}$ sIR. Full line is Eq. (3) fitted to the data points yielding a $K_{\mathrm{d}}=17 \pm 4 \mu \mathrm{M}$.

Table 2

Comparison of $K_{\mathrm{d}}$ values (in $\mu \mathrm{M} \pm \mathrm{SEM}$ ) of EO and ER binding to insulin receptor constructs obtained by displacement assay and spectroscopic measurements

\begin{tabular}{lccc}
\hline & Displacement & UV-vis & Fluorescence \\
\hline EO + sIR & $40 \pm 4^{\mathrm{a}}$ & $24 \pm 12^{\mathrm{b}}$ & - \\
$\mathrm{ER}+\mathrm{sIR}$ & $11 \pm 1^{\mathrm{a}}$ & - & $17 \pm 4^{\mathrm{c}}$ \\
$\mathrm{ER}+\mathrm{mIR}$ & - & $11 \pm 6^{\mathrm{a}}$ & $30 \pm 8^{\mathrm{a}}$ \\
$\mathrm{ER}+$ mIR & - & - & $22 \pm 8^{\mathrm{a}}$ \\
$\quad+$ insulin & & & \\
${ }^{\mathrm{a}}$ See [41]. & & & \\
${ }^{\mathrm{b}}$ See Fig. 6. & & & \\
${ }^{\mathrm{c}}$ See Fig. 7. & & &
\end{tabular}

(Fig. 7A). Using this quench to determine the affinity by fitting to Eq. (3) results in a $K_{\mathrm{d}}=17 \pm 4 \mu \mathrm{M}$, see Fig. 7B. This value is comparable to the $11 \pm 1 \mu \mathrm{M}$ obtained in the receptor binding assay.

Once the correlation between $K_{\mathrm{d}}$ values obtained by tracer-based receptor binding assays and by UVvis- and fluorescence spectroscopies was established, further spectroscopic characterization was performed using mIR preparations. Similar spectroscopic signatures of binding are observed for binding of ER to mIR using both UV-vis and fluorescence, and $K_{\mathrm{d}}$ values are obtained which are still comparable with those obtained for ER displacement of insulin from sIRFc. See Table 2 for a comparison of values obtained for EO and ER binding to sIR and mIR determined by the various assays and spectroscopies.

\subsubsection{NMR investigations of EO-mIR interaction}

By comparing ${ }^{1} \mathrm{H} 2 \mathrm{D}$ nuclear Overhauser effect (NOE) NMR spectra of $2 \mathrm{mM}$ EO in the absence and presence of $10 \mu \mathrm{M} \mathrm{mIR}$, it is observed that mIR induces transferred NOE cross peaks in the EO spectrum, see Fig. 8: The spectrum of EO alone has few, mostly negative cross peaks. In contrast, the presence of a low $[\mathrm{mIR}]$ results in the appearance of more cross peaks, all of which are relatively intense and of positive sign as the diagonal signals. Also the ripples in the spectrum of EO alone, caused by a truncation effect due to narrow line widths of the signals, are gone in the presence of mIR indicating broader signals. Both observations are consequences of the slower tumbling of the EO molecules when bound to $\mathrm{mIR}$, and hence confirm the binding of EO to mIR. 


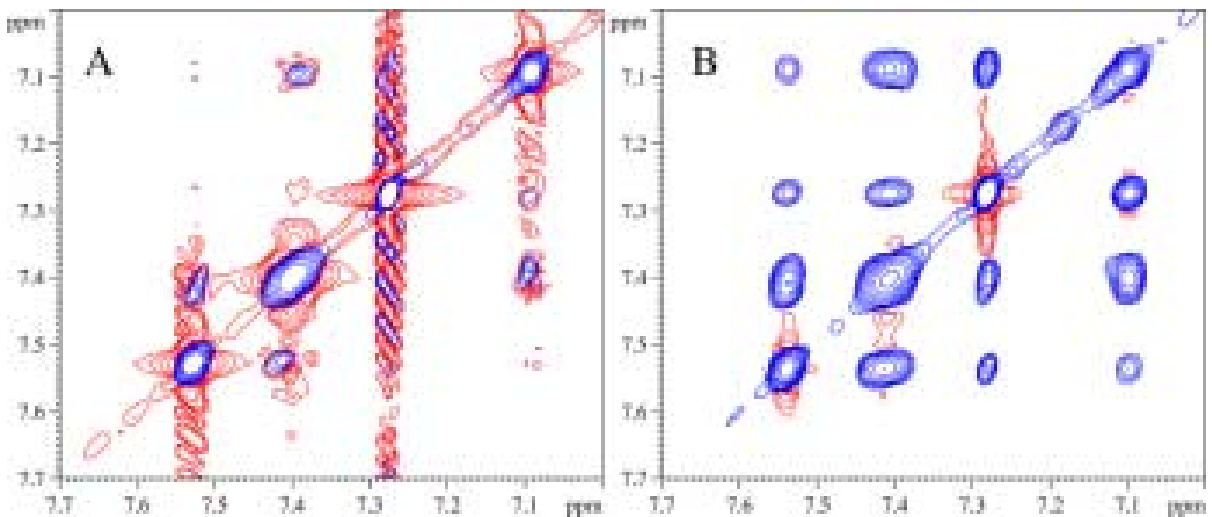

Fig. 8. Transferred NOESY cross peaks in the EO spectrum induced by mIR. The ${ }^{1} \mathrm{H} 2 \mathrm{D}$ NOESY spectrum of $2 \mathrm{mM} E O$ in the absence (A) and presence of $10 \mu \mathrm{M}$ mIR (B). Negative contours are colored red, positive are colored blue. Reprinted with permission from Biochemistry 40 (2001), 13 520-13 528. Copyright 2001 American Chemical Society.

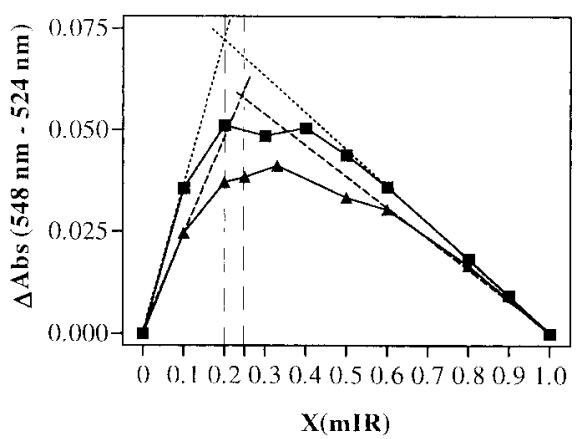

Fig. 9. Job plot of the ER-mIR interaction. Total concentration of ER and mIR is $86 \mu \mathrm{M}$. Depicted is the difference between the two extremes at 548 and $524 \mathrm{~nm}$ in the difference absorption spectra between samples containing mIR and ER and samples only containing ER versus the mole fraction of mIR. Samples without insulin are shown as (ם) and with $80 \mu \mathrm{M}$ insulin HisB10Asp ProB28Asp present as $(\boldsymbol{\Lambda})$. Tangents to the two curves are drawn through $x=0$ and $x=1$. Indicated by vertical dashed lines are $x=0.25$ (equals three ligands bound by each receptor molecule) and $x=0.2$ (equals four ligands bound). Reprinted with permission from Biochemistry 40 (2001), 13 520-13 528. Copyright 2001 American Chemical Society.

\subsubsection{Stoichiometry of ER-mIR interaction}

Although the $K_{\mathrm{d}}$ values obtained by spectroscopic measurements were similar to those initially found using a receptor binding assay, and the binding was shown to be reversible, it was impossible to displace the receptor induced spectroscopic changes by addition of insulin or by preincubating the receptor with insulin before the ligand titration. This was unexpected, as the small molecule ligand displacement of insulin from sIRFc apparently fitted to a one-site binding model.

This could be explained by the existence of multiple small-molecule binding sites on the receptors with similar binding constants. Therefore, the binding stoichiometry of the interaction between ER and mIR both in the absence and presence of insulin was determined using the continuous variation method $[15,19]$ and difference absorption spectroscopy. The Job plots of the ER-mIR interaction are shown in Fig. 9. The optimum of the Job plot is shifted to lower mole fraction of mIR than 0.5 both in the absence and presence of saturating insulin concentration, clearly indicating more than one molecule of ER bound per molecule mIR. That is, four ER molecules are bound by mIR, while in the presence of insulin the stoichiometry is reduced to three. The shift in the optimum towards higher mIR mole 
fraction and the smaller $\Delta \mathrm{Abs}$ signal in the presence of insulin are consistent with at least one bound ER molecule residing in or overlapping with the insulin binding site on mIR. This observation explains the apparent discrepancy between the receptor binding assay, where displacement of hormone from the intrinsic binding site is observed, and the spectroscopic titrations, where all binding phenomena with affinities in the same range are observed.

\section{Discussion}

The two examples illustrate the productive use of spectroscopy to study ligand binding to IR. First, the binding of the fluorescent B29NBD-insulin to mIR elucidated the reaction mechanism, which involves a binding event followed by a conformational change of the initially formed complex. Second, spectroscopic measurements revealed a more complex binding mode of small molecule ligands than assumed from standard biochemical receptor assays.

Conformational changes in the IR ectodomain (corresponding to sIR) upon insulin binding has been observed by CD spectroscopy [39]. These could be a consequence of the conformational changes in the smaller mIR construct which are described here. Fluorescence anisotropy changes upon insulin binding have been followed for the ectodomain [39] and for IR [26]. In both cases, however, these changes occur on much slower time scale than those reported here. The transmembrane segment in IR appears to play an important role in the transmission of global conformational changes upon insulin binding, as shown by solution studies [10].

For the homologous IGF-1 and the ectodomain of its receptor, overall on- and off-rates have been determined by Biacore techniques and are of similar size to those found in the present study. Furthermore, $\mathrm{CD}$ spectra of the ectodomain alone and in the presence of the hormone ligand were indicative of conformational changes induced by ligand binding [18].

The structure of the homologous minimized IGF-1 receptor (Fig. 1C) supports the proposed binding mechanism. As noted by Garrett et al. [12] the insulin molecule fits into the horseshoe-like notch, containing a number of residues important for binding ([12] and references herein). The binding mechanism then describes the fast binding of insulin into the notch area, followed by a slower conformational change that includes moving the $\mathrm{C}$-terminal part of the receptor containing the crucial 704-719 sequence relative to the notch (or vice versa) to complete high affinity complex formation.

Our data emphasize the importance of hormone induced conformational changes in activation of the holoreceptor.

The small molecule ligands binding to sIR and mIR are all antagonists and have no activating effect on IR. However, they constitute a new and important model system for spectroscopic characterization of small molecule ligands for IR.

Fluorescein and derivatives have been shown to bind to a number of different proteins $[5,6,13,14$, $29,33,42]$. Furthermore, the changes in UV-vis and fluorescence spectra are of similar nature as those observed when EO and ER bind to other proteins [20,25,34,43].

The transferred NOESY NMR experiment confirmed the binding of EO to mIR illustrating the application of such experiments and NMR line width broadening as a screening tool $[8,31,32]$. Furthermore, it validates $\mathrm{mIR}$ as a screening target in such experiments.

The apparent discrepancy between the one-site displacement of insulin by the small molecule ligands and the inability of insulin to displace the sIR and mIR induced changes in ligand spectra was intriguing. However, the Job plot revealed that mIR apparently has four binding sites for ER of which three are noncompetitive with the binding of insulin. All four binding sites must have very similar affinity towards ER 
since they appear as a single class as judged by the spectroscopic changes. Nevertheless, one of those sites then overlaps with the insulin binding site and give rise to the displacement of insulin from sIRFc.

In a recent study, a large number of molecules repeatedly found as inhibitor hits in virtual and highthroughput screens were examined. These included the ER derivative rose Bengal and tetraiodophenolphthalein which both displace insulin form sIRFc in a similar way as ER and EO [41]. The authors suggested that these so-called "promiscuous inhibitors" exhibit their inhibition by forming large micellelike aggregates which encapsulate the protein of interest. This hypothesis is based on a loss of inhibition when increasing the protein concentration ten-fold (even with 1000-fold increased inhibitor concentration), an increased inhibition after longer time incubation of protein with inhibitor, and the appearance of large particles detected by dynamic light scattering in a concentration range covering the EC50 values [30]. These interesting findings are most relevant to the fields of drug discovery and high-throughput screening.

The present spectroscopic results of binding of similar molecules to IR are unlikely to originate from a mechanism involving micellation of the ligands. There are magnitudes in difference between the sIRFc concentration used in the displacement receptor binding assay and the micromolar amounts used in various spectroscopic titrations. Although the binding assay equilibrates over night, the spectroscopic titrations are performed without incubation. Still, similar $K_{\mathrm{d}}$ values are determined in both binding assay as well as spectroscopic titrations. Indeed, all the spectroscopic measurements are based on changes in ligand spectra in the presence of receptor rather than inhibition of receptor function. Hence, the spectroscopic experiments complement the displacement receptor binding assay. If the ligands were assembled in large aggregates, they would have much slower tumbling times behaving as a large molecule and hence exhibit positive cross peaks in NOESY NMR spectra, as seen with suramin [37]. This only occurs in the presence of mIR (see Fig. 8), indicating no preformed ligand aggregates. Finally, the stoichiometry shows a small number of ligands bound to $\mathrm{mIR}$, and not the high ligand:receptor ratio expected if $\mathrm{mIR}$ was encapsulated by a larger ligand aggregate.

\section{Conclusion}

These two cases are examples on how spectroscopic studies of IR ligand binding can complement and detail classical biochemical receptor binding assays such as functional and displacement assays. Although structural description of the systems, when available, is of higher atomic detail, the present studies reflect the dynamics of binding reactions in solution.

\section{Acknowledgements}

We thank Ane M. Blom, Susan E. Danielsen, Anne-Marie Kolstrup for excellent technical assistance and Lene Drube, Else Jost Jensen and Claus Kristensen for supplying receptor preparations.

\section{References}

[1] A.J. Ablooglu, M. Frankel, E. Rusinova, J.B. Ross and R.A. Kohanski, Multiple activation loop conformations and their regulatory properties in the insulin receptor's kinase domain, J. Biol. Chem. 276 (2001), 46933-46940.

[2] A.S. Andersen, T. Kjeldsen, F.C. Wiberg, P.M. Christensen, J.S. Rasmussen, K. Norris, K.B. Møller and N.P. Møller, Changing the insulin receptor to possess insulin-like growth factor I ligand specificity, Biochemistry 29 (1990), 73637366. 
[3] E.N. Baker, T.L. Blundell, J.F. Cutfield, S.M. Cutfield, E.J. Dodson, G.G. Dodson, D.M. Hodgkin, R.E. Hubbard, N.W. Isaacs, C.D. Reynolds and K.S.N.V.N.M. Sakabe, The structure of $2 \mathrm{Zn}$ pig insulin crystals at 1.5 A resolution, Philos. Trans. R. Soc. Lond. B Biol. Sci. 319 (1988), 369-456.

[4] C.F. Bernasconi, Relaxation Kinetics, Academic Press, New York, 1976.

[5] L. Brand, J.R. Gohlke and D.S. Rao, Evidence for binding of rose bengal and anilinonaphthalenesulfonates at the active site regions of liver alcohol dehydrogenase, Biochemistry 6 (1967), 3510-3518.

[6] T. Chiba, Y. Sato and Y. Suzuki, Characterization of eosin 5-isothiocyanate binding site in band 3 protein of the human erythrocyte, Biochim. Biophys. Acta 897 (1987), 14-24.

[7] M.H. Cobb, B.C. Sang, R. Gonzalez, E. Goldsmith and L. Ellis, Autophosphorylation activates the soluble cytoplasmic domain of the insulin receptor in an intermolecular reaction, J. Biol. Chem. 264 (1989), 18 701-18 706.

[8] J. Fejzo, C.A. Lepre, J.W. Peng, G.W. Bemis, Ajay, M.A. Murcko and J.M. Moore, The SHAPES strategy: An NMR-based approach for lead generation in drug discovery, Chem. Biol. 6 (1999), 755-769.

[9] J.R. Flores-Riveros, E. Sibley, T. Kastelic and M.D. Lane, Substrate phosphorylation catalyzed by the insulin receptor tyrosine kinase. Kinetic correlation to autophosphorylation of specific sites in the beta subunit, J. Biol. Chem. 264 (1989), $21557-21572$.

[10] R.R. Flörke, K. Schnaith, W. Passlack, M. Wichert, L. Kuehn, M. Fabry, M. Federwisch and H. Reinauer, Hormonetriggered conformational changes within the insulin-receptor ectodomain: requirement for transmembrane anchors, Biochem. J. 360 (2001), 189-198.

[11] M. Frankel, S.M. Bishop, A.J. Ablooglu, Y.P. Han and R.A. Kohanski, Conformational changes in the activation loop of the insulin receptor's kinase domain, Protein Sci. 8 (1999), 2158-2165.

[12] T.P. Garrett, N.M. McKern, M. Lou, M.J. Frenkel, J.D. Bentley, G.O. Lovrecz, T.C. Elleman, L.J. Cosgrove and C.W. Ward, Crystal structure of the first three domains of the type-1 insulin-like growth factor receptor, Nature 394 (1998), 395-399.

[13] C. Gatto and M.A. Milanick, Inhibition of the red blood cell calcium pump by eosin and other fluorescein analogues, $A m$. J. Physiol. 264 (1993), C1577-C1586.

[14] S.C. Hopkins, R.D. Vale and I.D. Kuntz, Inhibitors of kinesin activity from structure-based computer screening, Biochemistry 2000 (2000), 2805-2814.

[15] C.Y. Huang, Determination of binding stoichiometry by the continuous variation method: The Job plot, Methods Enzymol. 87 (1982), 509-525.

[16] S.R. Hubbard, Crystal structure of the activated insulin receptor tyrosine kinase in complex with peptide substrate and ATP analog, EMBO J. 16 (1997), 5572-5581.

[17] S.R. Hubbard, L. Wei, L. Ellis and W.A. Hendrickson, Crystal structure of the tyrosine kinase domain of the human insulin receptor, Nature 372 (1994), 746-754.

[18] M. Jansson, D. Hallen, H. Koho, G. Andersson, L. Berghard, J. Heidrich, E. Nyberg, M. Uhlen, J. Kordel and B. Nilsson, Characterization of ligand binding of a soluble human insulin-like growth factor I receptor variant suggests a ligandinduced conformational change, J. Biol. Chem. 272 (1997), 8189-8197.

[19] P. Job, Ann. Chim. 9 (1928), 113-134.

[20] I. Kalousek, D. Jandova and Z. Vodrazka, The interaction of human hemoglobin with erythrosin. Comparison of hemoglobins with variously liganded heme groups, Eur. J. Biochem. 86 (1978), 417-422.

[21] P.J. Kraulis, Molscript - A program to produce both detailed and schematic plots of protein structures, J. App. Crystallogr. 24 (1991), 946-950.

[22] C. Kristensen, A.S. Andersen, S. Østergaard, P.H. Hansen, and J. Brandt, Functional reconstitution of insulin receptor binding site from non-binding receptor fragments, J. Biol. Chem. 277 (2002), 18 340-18 345.

[23] C. Kristensen, F.C. Wiberg and A.S. Andersen, Specificity of insulin and insulin-like growth factor I receptors investigated using chimeric mini-receptors - Role of C-teriminal of receptor alpha subunit, J. Biol. Chem. 274 (1999), 37 351-37 356.

[24] C. Kristensen, F.C. Wiberg, L. Schaffer and A.S. Andersen, Expression and characterization of a 70-kDa fragment of the insulin receptor that binds insulin. Minimizing ligand binding domain of the insulin receptor, J. Biol. Chem. 273 (1998), $17780-17786$.

[25] D.J.R. Laurence, A study of the adsorption of dyes on bovine serum albumine by the method of polarization of fluorescence, Biochem. J. 51 (1952), 168-180.

[26] J. Lee, P.F. Pilch, S.E. Shoelson and S.F. Scarlata, Conformational changes of the insulin receptor upon insulin binding and activation as monitored by fluorescence spectroscopy, Biochemistry 36 (1997), 2701-2708.

[27] S. Ludvigsen, H.B. Olsen and N.C. Kaarsholm, A structural switch in a mutant insulin exposes key residues for receptor binding, J. Mol. Biol. 279 (1998), 1-7.

[28] S. Ludvigsen, M. Roy, H. Thøgersen and N.C. Kaarsholm, High-resolution structure of an engineered biologically potent insulin monomer, B16 Tyr $\rightarrow$ His, as determined by nuclear magnetic resonance spectroscopy, Biochemistry 33 (1994), 7998-8006. 
[29] E. Majima, N. Yamaguchi, H. Chuman, Y. Shinohara, M. Ishida, S. Goto and H. Terada, Binding of the fluorescein derivative eosin Y to the mitochondrial ADP/ATP carrier: characterization of the adenine nucleotide binding site, Biochemistry 37 (1998), 424-432.

[30] S.L. McGovern, E. Caselli, N. Grigorieff and B.K. Shoichet, A common mechanism underlying promiscuous inhibitors from virtual and high-throughput screening, J. Med. Chem. 45 (2002), 1712-1722.

[31] B. Meyer, T. Weimar, and T. Peters, Screening mixtures for biological activity by NMR, Eur. J. Biochem. 246 (1997), 705-709.

[32] J.M. Moore, NMR techniques for characterization of ligand binding: Utility for lead generation and optimization in drug design, Biopolymers 51 (1999), 221-243.

[33] G.G. Neslund, J.E. Miara, J.J. Kang and A.S. Dahms, Specific interactions of xanthene dyes with nucleotide-binding sites of membrane energy-transducing enzymes and carriers, Curr. Top. Cell. Regul. 24 (1984), 447-469.

[34] N.G. Oikonomakos, T.G. Sotiroudis and A.E. Evangelopoulos, Interaction of phosphorylase b with eosin. Influence of substrate and effectors on eosin-enzyme complexes, Biochem. J. 181 (1979), 309-320.

[35] H.B. Olsen, S. Ludvigsen and N.C. Kaarsholm, Solution structure of an engineered insulin monomer at neutral $\mathrm{pH}$, Biochemistry 35 (1996), 8836-8845.

[36] H.B. Olsen, S. Ludvigsen and N.C. Kaarsholm, The relationship between insulin bioactivity and structure in the $\mathrm{NH}_{2}-$ terminal A-chain helix, J. Mol. Biol. 284 (1998), 477-488.

[37] T. Polenova, T. Iwashita, A.G. Palmer and A.E. McDermott, Conformation of the trypanocidal pharmaceutical suramin in its free and bound forms: transferred nuclear Overhauser studies, Biochemistry 36 (1997), 14 202-14 217.

[38] O.M. Rosen, R. Herrera, Y. Olowe, L.M. Petruzzelli and M.H. Cobb, Phosphorylation activates the insulin receptor tyrosine protein kinase, Proc. Natl. Acad. Sci. USA 80 (1983), 3237-3240.

[39] E.M. Schaefer, H.P. Erickson, M. Federwisch, A. Wollmer and L. Ellis, Structural organization of the human insulin receptor ectodomain, J. Biol. Chem. 267 (1992), 23 393-23 402.

[40] M. Schlein, S. Havelund, C. Kristensen, M.F. Dunn and N.C. Kaarsholm, Ligand-induced conformational change in the minimized insulin receptor, J. Mol. Biol. 303 (2000), 161-169.

[41] M. Schlein, S. Ludvigsen, H.B. Olsen, A.S. Andersen, G.M. Danielsen and N.C. Kaarsholm, Properties of small molecules affecting insulin receptor function, Biochemistry 40 (2001), 13 520-13 528.

[42] J.C. Skou and M. Esmann, Eosin, a fluorescent probe of ATP binding to the $\left(\mathrm{Na}^{+}+\mathrm{K}^{+}\right)$- ATPase, Biochim. Biophys. Acta 647 (1981), 232-240.

[43] L.L. Somerville and F.A. Quiocho, The interaction of tetraiodofluorescein with creatine kinase, Biochim. Biophys. Acta 481 (1977), 493-499.

[44] L.G. Sparrow, N.M. McKern, J.J. Gorman, , P.M. Strike, C.P. Robinson, J.D. Bentley and C.W. Ward, The disulfide bonds in the C-terminal domains of the human insulin receptor ectodomain, J. Biol. Chem. 272 (1997), 29460-29 467.

[45] A. Ullrich, J.R. Bell, E.Y. Chen, R. Herrera, L.M. Petruzzelli, T.J. Dull, A. Gray, L. Coussens, Y.C. Liao, M. Tsubokawa and A. Manson, Human insulin receptor and its relationship to the tyrosine kinase family of oncogenes, Nature 313 (1985), $756-761$.

[46] M.F. White and C.R. Kahn, The insulin signaling system, J. Biol. Chem. 269 (1994), 1-4.

[47] G. Williams and J.C. Pickup, Handbook of Diabetes, Blackwell, Oxford, 1999. 


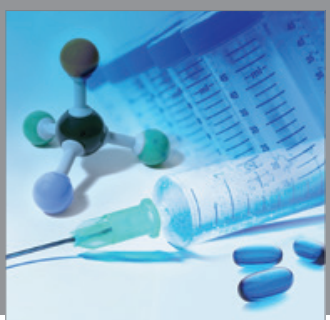

International Journal of

Medicinal Chemistry

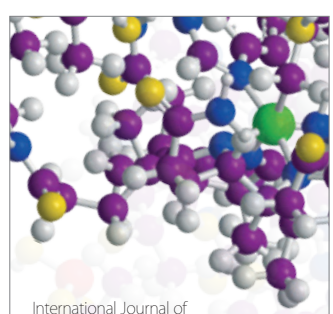

Carbohydrate Chemistry

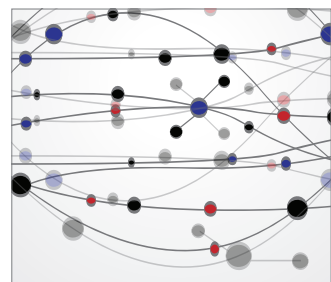

The Scientific World Journal
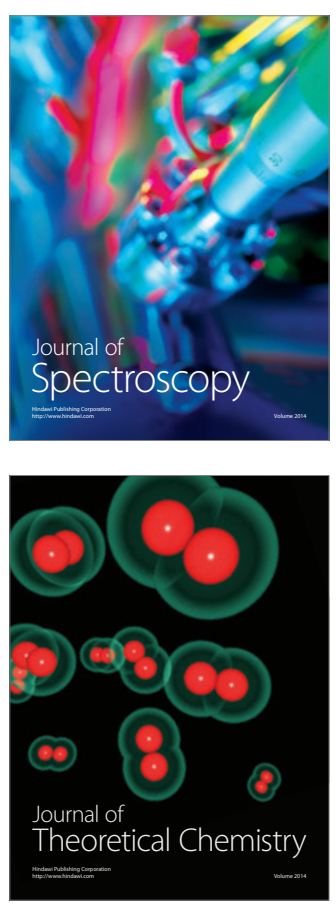
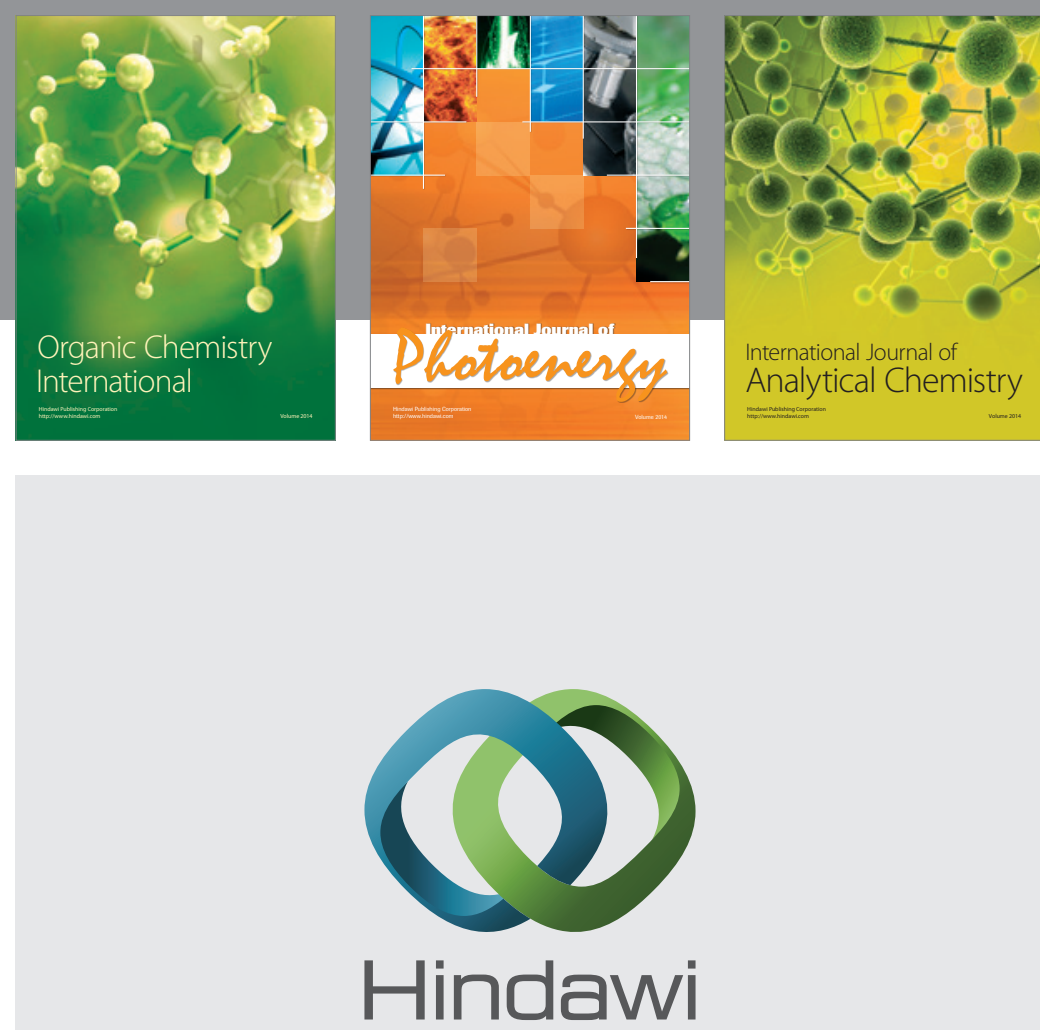

Submit your manuscripts at

http://www.hindawi.com
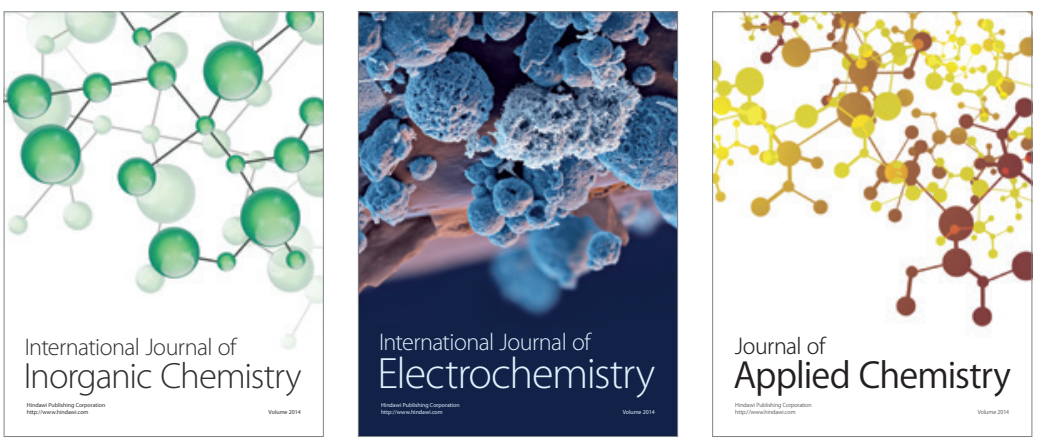

Journal of

Applied Chemistry
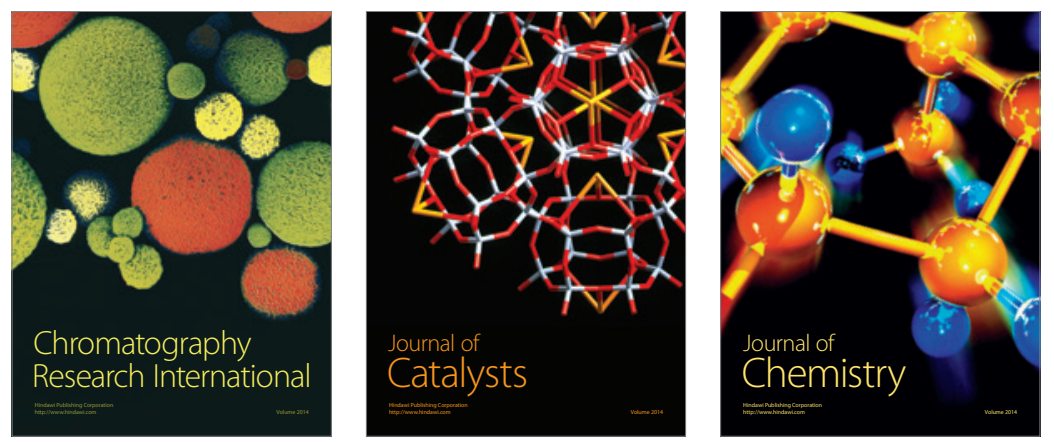
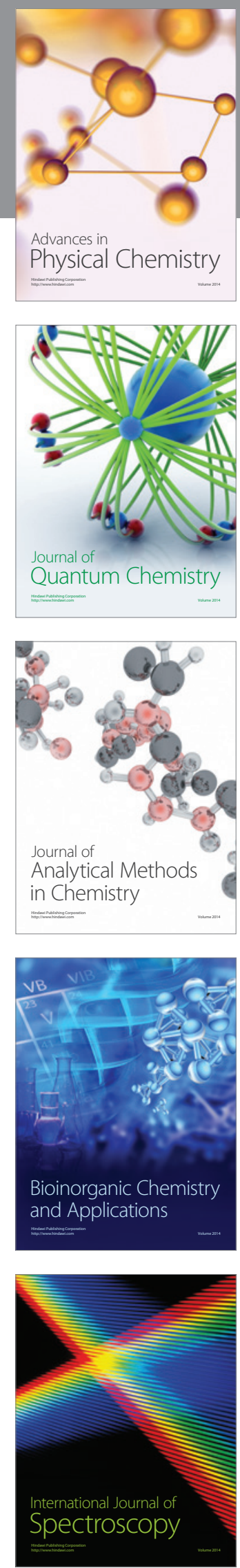